Wright State University

CORE Scholar

6-1-2009

\title{
Effects of Electron-Irradiation on Electrical Properties of AlGaN/ GaN Schottky Barrier Diodes
}

\author{
Z-Q. Fang \\ Gary C. Farlow \\ Wright State University - Main Campus, gary.farlow@wright.edu \\ B. Claflin \\ David C. Look \\ Wright State University - Main Campus, david.look@wright.edu \\ D. S. Green
}

Follow this and additional works at: https://corescholar.libraries.wright.edu/physics

Part of the Physics Commons

\section{Repository Citation}

Fang, Z., Farlow, G. C., Claflin, B., Look, D. C., \& Green, D. S. (2009). Effects of Electron-Irradiation on Electrical Properties of AIGaN/GaN Schottky Barrier Diodes. Journal of Applied Physics, 105 (12), 123704. https://corescholar.libraries.wright.edu/physics/164

This Article is brought to you for free and open access by the Physics at CORE Scholar. It has been accepted for inclusion in Physics Faculty Publications by an authorized administrator of CORE Scholar. For more information, please contact library-corescholar@wright.edu. 


\title{
Effects of electron-irradiation on electrical properties of AIGaN/GaN Schottky barrier diodes
}

\author{
Z.-Q. Fang, ${ }^{1, a)}$ G. C. Farlow, ${ }^{2}$ B. Claflin, ${ }^{1}$ D. C. Look, ${ }^{1}$ and D. S. Green ${ }^{3}$ \\ ${ }^{1}$ Semiconductor Research Center, Wright State University, Dayton, Ohio 45435, USA and Materials and \\ Manufacturing Directorate, Air Force Research Laboratory, Wright Patterson AFB, Ohio 45433, \\ USA \\ ${ }_{2}^{2}$ Department of Physics, Wright State University, Dayton, Ohio 45435, USA \\ ${ }^{3}$ Aerospace and Defense, RF Micro Devices, Charlotte, North Carolina 28269, USA
}

(Received 7 April 2009; accepted 12 May 2009; published online 17 June 2009)

\begin{abstract}
Effects of $1 \mathrm{MeV}$ electron-irradiation at room temperature on the electrical properties of AlGaN/ GaN heterostructures, including leakage currents, threshold voltages, and electron traps, have been investigated using Schottky barrier diodes (SBDs) fabricated on the AlGaN. The SBDs, before and after the irradiation with a dose of $5 \times 10^{15} \mathrm{~cm}^{-2}$, were characterized by temperature dependent current-voltage and capacitance-voltage measurements and deep level transient spectroscopy. It is found that the irradiation causes (i) significant increase in leakage currents, dominated by tunneling conduction, at both reverse and low-forward biases; (ii) a clear negative shift in threshold voltage in the pinch-off region; and (iii) creation of traps $A_{e}(\sim 1.1 \mathrm{eV})$ or $A_{2}(1.2 \mathrm{eV})$ and $E_{e}(0.09 \mathrm{eV})$ in the $\mathrm{GaN}$ buffer and $\mathrm{AlGaN}$ regions. The irradiation-induced traps can be used to account for the increase in leakage currents and shift in threshold voltage. However, as compared to traps $A_{2}(1.2 \mathrm{eV})$ and $E(0.13 \mathrm{eV})$ induced in thick GaN layers by electron-irradiation, the irradiation-induced traps in the $\mathrm{AlGaN} / \mathrm{GaN}$ heterostructures show some changes in activation energy and electron capture behavior.
\end{abstract}

(C) 2009 American Institute of Physics. [DOI: 10.1063/1.3151952]

\section{INTRODUCTION}

The AlGaN/GaN heterostructure field effect transistor (HFET) is considered to be the key device for the next generation of high-power and high-frequency electronics. Effect of radiation damage on device performance is a major concern for space applications of GaN-based HFETs because of high energy electron and proton exposure in the Earth's radiation belt. Several previous studies were conducted on the effects of irradiation on HFET devices. In 2004, Hu et al. ${ }^{1}$ reported the energy dependence of proton-induced degradation and recovery of AlGaN/GaN HFETs. In 2008, Sonia et $a l .^{2}$ studied the effect of $2 \mathrm{MeV}$ proton-irradiation on HFETs and found that irradiation with protons up to fluences of $10^{13} \mathrm{~cm}^{-2}$ did not degrade the operation of the devices. In 2007, low-energy ( $0.45 \mathrm{MeV})$ electron-irradiation (EI) effects on HFETs were investigated at low temperature $(\sim 85 \mathrm{~K})$ by McClory et $a l^{3}{ }^{3}$ In that study, increases in the drain and gate currents and a shift in the threshold voltage found at low temperatures were discussed in terms of positive charge buildup and the trap formation in the AlGaN layer (or near the interface). EI effects can be also studied using AlGaN/GaN Schottky barrier diodes (SBDs); however, very few such works have been reported. Recently, Polyakov et al. ${ }^{4}$ reported effects of $10 \mathrm{MeV}$ EI on $\mathrm{AlGaN} / \mathrm{GaN}$ and AlN/GaN heterostructures grown by molecular beam epitaxy (MBE) and found an increase in the GaN buffer resistivity due to compensation by radiation defects with levels at $\sim E_{c}-1 \mathrm{eV}$. In this letter, we present effects of $1 \mathrm{MeV}$ EI on

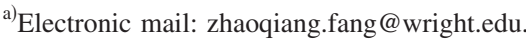

electrical properties of $\mathrm{AlGaN} / \mathrm{GaN}$ SBDs grown by metalorganic vapor phase epitaxy (MOVPE). Temperature dependent current-voltage $(I-V)$, capacitance-voltage $(C-V)$, and deep level transient spectroscopy (DLTS) were used to study significant EI-induced changes in leakage current, threshold voltage, and electron traps.

\section{EXPERIMENTAL}

The structure used in this study consisted of an AlN nucleation layer, an unintentionally doped $\mathrm{GaN}$ buffer layer ( $\sim 2 \mathrm{~mm}$ thick), and an unintentionally doped AlGaN layer ( $\sim 225 \AA$ thick), with an $\mathrm{Al}$ mole fraction of $25 \%$ grown by MOVPE on a SiC substrate. (For details of the nucleation layer, see a study about the impact of AlN nucleation conditions on the density and character of threading dislocations emanating from the on $\mathrm{SiC}$ substrates. ${ }^{5}$ ) It is also possible that carbon could be incorporated into the unintentionally doped $\mathrm{GaN}$ buffer layer, making it highly resistive, but we have no direct evidence of that fact. Schottky dots of $130 \mu \mathrm{m}$ in diameter and ring-shaped Ohmic contacts surrounding the dots were fabricated on the surface using $\mathrm{Ni} / \mathrm{Au}$ and $\mathrm{Ti} / \mathrm{Al} / \mathrm{Ni} / \mathrm{Au}$ metallizations, respectively. The region between the Schottky dot and Ohmic contact was passivated with $\mathrm{Si}_{3} \mathrm{~N}_{4}$. Irradiation by $1 \mathrm{MeV}$ electrons with a dose of $5 \times 10^{15} \mathrm{~cm}^{-2}$ was performed at room temperature using a Van de Graaff accelerator. The $C-V$ and DLTS measurements were conducted with an Accent DL8000 spectrometer operated at a frequency of $1 \mathrm{MHz}$ for the capacitance measurements. DLTS spectra were obtained from Fourier transforms of capacitance transients as the temperature was swept from 


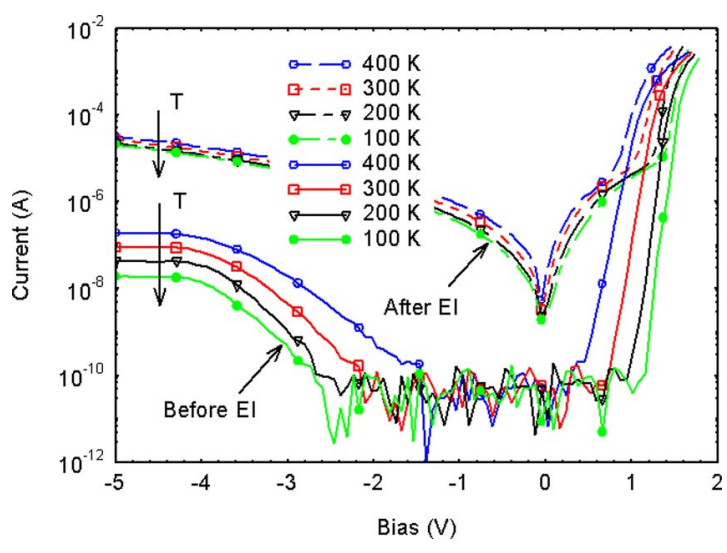

FIG. 1. (Color online) Temperature dependent $I-V$ characteristics measured before and after $1 \mathrm{MeV}$ EI.

200 to $450 \mathrm{~K}$ (using a $\mathrm{LN}_{2}$-cryostat) or from 30 to $300 \mathrm{~K}$ (using a LHe-cryostat). The $I-V$ measurements were carried out with a current amplifier in the spectrometer. In the measurements of the temperature dependent $I-V$ and $C$ - $V$ characteristics, the SBDs were first warmed to $450 \mathrm{~K}$ to avoid illumination effects from a microscope light used during sample mounting and then cooled from 450 to $100 \mathrm{~K}$ in $50 \mathrm{~K}$ steps with about 3 min cooling time between the steps.

\section{RESULTS AND DISCUSSION}

The $I-V-T$ characteristics, measured at temperatures from 400 to $100 \mathrm{~K}$ before and after $1 \mathrm{MeV} \mathrm{EI}$, are presented in Fig. 1. From the $I-V-T$ curves before EI, we see that (i) the forward currents versus bias show a good exponential relationship at low biases and can be described by thermionic emission (e.g., ideality factor is 1.45 and Schottky barrier height is $1.1 \mathrm{eV}$ at $300 \mathrm{~K}$ ); (ii) as compared to the forward currents, the reverse leakage currents are not strongly temperature dependent, indicating tunneling conduction; and (iii) the reverse currents at $V_{R}<-4 \mathrm{~V}$ are quite low (1.5 $\times 10^{-3} \mathrm{~A} / \mathrm{cm}^{2}$ at $\left.400 \mathrm{~K}\right)$ and nearly constant due to complete pinch-off of the two-dimensional electron gas (2DEG) (compare Fig. 2). However, from the $I-V$ - $T$ curves after EI, we find that (i) the EI induces a significant increase in leak-

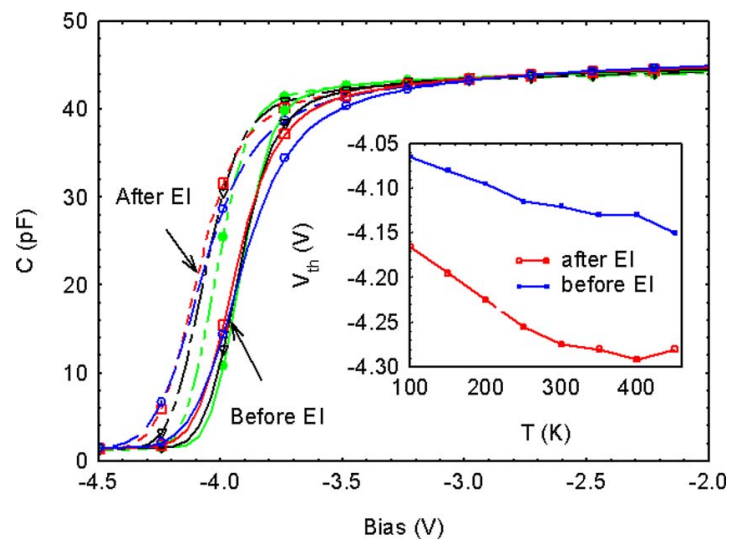

FIG. 2. (Color online) Temperature dependent $C$ - $V$ characteristics measured before and after EI [curves marked with open circle $(400 \mathrm{~K})$, square (300 $\mathrm{K})$, triangle $(200 \mathrm{~K})$, and solid circle $(100 \mathrm{~K})]$. Inset shows the temperature dependence of $V_{\text {th }}$. age currents under both reverse and low-forward biases (by more than two orders at $U_{R}=-5 \mathrm{~V}$ ) and (ii) the EI-induced leakage currents show strong electric-field dependence and much less temperature dependence, indicating that tunneling conduction dominates.

The mechanism responsible for leakage currents in AlGaN/GaN SBDs has been extensively investigated worldwide. Zhang et al. ${ }^{6}$ confirmed the role of conductive dislocations in leakage currents for both $\mathrm{GaN}$ and $\mathrm{AlGaN} / \mathrm{GaN}$ structures, grown by MBE, and suggested that the key carrier tunneling transport process is emission of electrons from a trap state near the metal-semiconductor interface into a continuum of states associated with each conductive dislocation (designated "dislocation model" here). Hashizume et al. ${ }^{7}$ proposed a thin surface barrier (TSB) model in which the width of the Schottky barrier on $\mathrm{GaN}$ or $\mathrm{AlGaN}$, grown by MOVPE, is reduced due to the presence of unintentional surface-defect donors, probably related to nitrogen vacancies. This model assumes that electrons can tunnel through the thin barrier in both forward and reverse directions, by thermionic field-emission or field-emission alone, depending on the temperature. In addition, Kotani et al. ${ }^{8}$ suggested a mechanism of surface conduction in the vicinity of Schottky gates on $\mathrm{AlGaN} / \mathrm{GaN}$ heterostructures since they found that the lateral surface leakage current showed two-dimensional variable-range hopping through high-density surface states on $\mathrm{AlGaN}$ and could be significantly reduced with $\mathrm{SiN}_{x}$ passivation.

We find that defects related to nitrogen vacancies and possibly nitrogen interstitials can be produced in both $\mathrm{GaN}$ buffer and AlGaN cap layers, as well as on the AlGaN surface by $1 \mathrm{MeV}$ EI. At present, we have no evidence for the dislocation density increasing with this EI. It seems that the TSB model is better than the dislocation model to account for the EI-induced leakage currents. The intentionally induced defects under the Schottky contact and on the surface of AlGaN can facilitate electron tunneling through the TSB, resulting in the increase in leakage current. Actually, we also observed extra leakage currents, higher than the leakage currents (after EI) shown in Fig. 1, when the temperature was first raised from 300 to $450 \mathrm{~K}$. The additional leakage currents disappeared when the temperature was reduced from $450 \mathrm{~K}$ and might be related to surface conduction due to adsorption.

The $C-V-T$ characteristics before and after $1 \mathrm{MeV} \mathrm{EI}$, measured at temperatures of 400-100 K, are shown in Fig. 2. We observe that (i) the threshold voltage $\left(V_{\text {th }}\right)$ determined from each $C-V$ curve in the pinch-off region for the SBD, either before or after EI, increases to a less negative value as the temperature decreases from 400 to $100 \mathrm{~K}$; (ii) as shown in the inset of Fig. 2, the rate of change in $V_{\text {th }}$ with temperature at $T<300 \mathrm{~K}$ is lower before $\mathrm{EI}\left(\Delta V_{\mathrm{th}}=-0.28 \mathrm{mV} /{ }^{\circ} \mathrm{C}\right)$ than after $\operatorname{EI}\left(\Delta V_{\mathrm{th}}=-0.55 \mathrm{mV} /{ }^{\circ} \mathrm{C}\right)$; and (iii) the EI causes a negative shift in the $V_{\text {th }}$ 's, with a shift of $-0.16 \mathrm{~V}$ at $300 \mathrm{~K}$. Interestingly, the EI did not change the carrier concentration of the 2DEG $\left(N_{s}\right)$, which is $7.5 \times 10^{19} \mathrm{~cm}^{-3}$ at $300 \mathrm{~K}$ (determined from $C-V$ ), but produced a shift in the 2DEG peak depth by about $0.2 \mathrm{~nm}$. According to Kordoš et al., ${ }^{9}$ the threshold voltage can be described as $V_{\mathrm{th}}=\Phi_{b}-\Delta E_{c}$ 


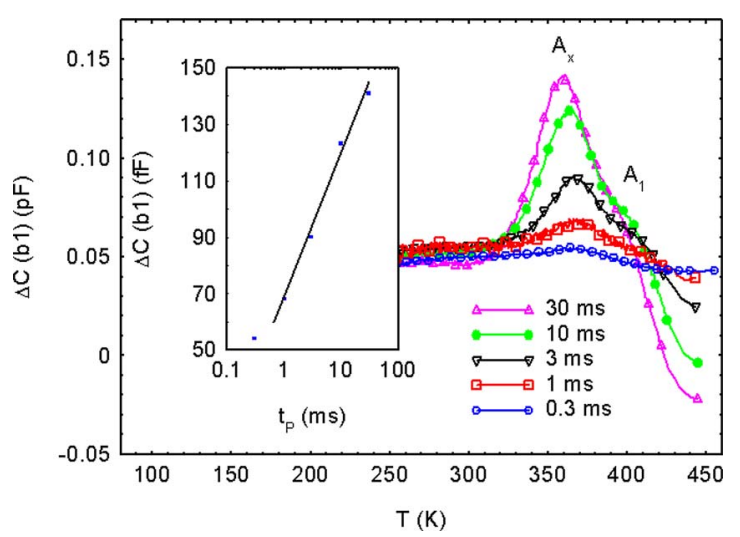

FIG. 3. (Color online) DLTS spectra measured as a function of filling pulse width $t_{P}$ for the SBD before EI (inset shows the logarithmic dependence of DLTS signal with $t_{P}$ ). Conditions: $U_{R}=-3.95 \mathrm{~V}, U_{P}=2 \mathrm{~V}, T_{W}=20.5 \mathrm{~ms}$, and $t_{P}=0.3-30 \mathrm{~ms}$.

- $\left(q N_{\text {tot }} d / 2 \varepsilon_{r} \varepsilon_{0}\right)$, where $\Phi_{b}$ is the barrier height, $\Delta E_{c}$ is the conduction band discontinuity, $d$ is the barrier-channel distance, and $\varepsilon_{r} \varepsilon_{0}$ is the dielectric constant. The total carrier density $N_{\text {tot }}$ is a sum of the densities of shallow carriers and carrier traps, $N_{\text {tot }}=N_{s}+N_{t}$. In Ref. 9, a temperature dependence of $V_{\text {th }}$ from 20 to $350{ }^{\circ} \mathrm{C}$ with a rate slope of $\Delta V_{\text {th }}=$ $-1.6 \mathrm{mV} /{ }^{\circ} \mathrm{C}$ was reported for AlGaN/GaN HFETs grown on sapphire. Based on their analysis of $\Delta V_{\text {th }}$, which can be expressed as $\Delta V_{\mathrm{th}}=q N_{T} d / 2 \varepsilon_{r} \varepsilon_{0}$, and assuming that the other terms have a much weaker temperature dependence, the authors attributed the observed changes to a trap $(\sim 0.2 \mathrm{eV})$ that is probably related to nitrogen vacancies near the surface of the $\mathrm{AlGaN}$ layer. Although the temperature range used in our study and the values of $\Delta V_{\text {th }}$ obtained are quite different from those reported in Ref. 9, we believe that the temperature dependence of $V_{\text {th }}$ is related to the presence of surface traps in the AlGaN since the negative shift in $V_{\text {th }}$ and the increase in $\left|\Delta V_{\text {th }}\right|$ after EI can be explained by EI-enhanced $N_{t}$, as described below.

Figure 3 presents DLTS spectra measured as a function of filling pulse width $\left(t_{P}\right)$ for the SBD before irradiation. Trap $A_{x}$ with activation energy of $0.9 \mathrm{eV}$ is found to be the dominant trap, and trap $A_{1}$ can be observed as a hightemperature shoulder on $A_{x}$. As shown in the inset, the DLTS signal of trap $A_{x}$ exhibits a logarithmic dependence with $t_{P}$, which indicates that it is associated with extended defects, such as threading dislocations. ${ }^{10,11}$ In our laboratory, traps in $\mathrm{AlGaN} / \mathrm{GaN} / \mathrm{SiC}$ heterostructures, grown by MOVPE, have been previously studied, and a dominant trap $A_{1}(1.0 \mathrm{eV})$, associated with threading dislocations, and a smaller trap $A_{2}$ were reported. ${ }^{11}$ Observations of different dominant traps are thought to be related to differences in the growth conditions for these heterostructures. Now, we compare DLTS results for the SBD before and after the EI. DLTS spectra at high temperatures from 200 to $450 \mathrm{~K}$, measured at reverse bias of $U_{R}=-3.95$ or $-4.05 \mathrm{~V}$ and filling pulse height of $U_{P}=2 \mathrm{~V}$, are shown in Fig. 4(a). Because of the shift in $V_{\text {th }}$, a more negative value of $V_{\text {th }}$ (i.e., $-4.05 \mathrm{~V}$ ) was used for the SBD after EI. From the figure, we see that (i) before EI, there exist three traps, $A_{x}, A_{1}$, and $A_{2}$ (which become observable in the spectrum due to the use of a longer $T_{W}$ ); and (ii) after EI,
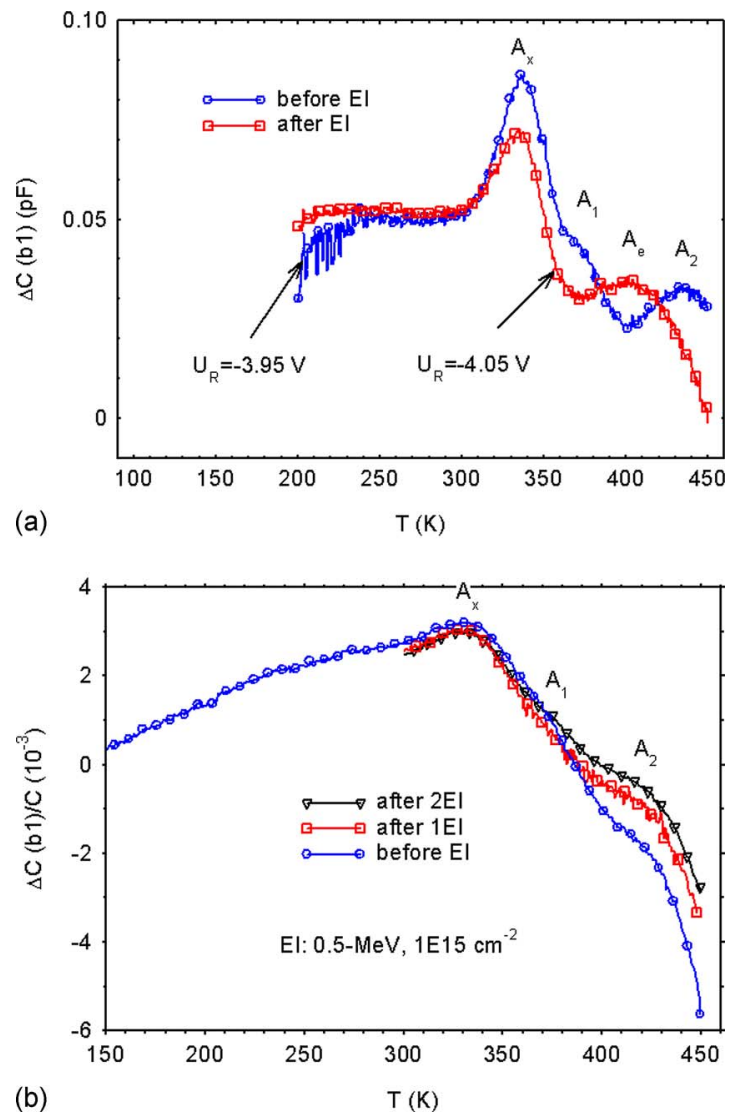

FIG. 4. (Color online) (a) DLTS spectra measured before and after EI for the tested AlGaN/GaN SBD. Conditions: $U_{P}=2 \mathrm{~V}, T_{W}=205 \mathrm{~ms}$, and $t_{P}$ $=30 \mathrm{~ms}$. (b) DLTS spectra measured before and after consecutive EIs for the control AlGaN/GaN SBD. Conditions: $U_{R}=-4 \mathrm{~V}, U_{P}=1 \mathrm{~V}, T_{W}$ $=205 \mathrm{~ms}$, and $t_{P}=30 \mathrm{~ms}$.

there are only two traps, $A_{x}$ and $A_{e}$. Trap $A_{x}$ is slightly reduced in magnitude, and it seems that the two traps $A_{1}$ and $A_{2}$ may join to form a new $\operatorname{trap} A_{e}$, with an estimated activation energy of $\sim 1.1 \mathrm{eV}$. This observation differs from that of our earlier studies on $\mathrm{GaN}$ in which only trap $A_{2}$ increased with EI. There we reported that either 0.4 or $1 \mathrm{MeV}$ EI creates a $V_{N}$-related trap $E(0.16 \mathrm{eV})$ and another trap $A_{2}(1.2 \mathrm{eV})$, possibly related to $N_{I}$, in thick $\mathrm{GaN}$ layers grown by hydride vapor phase epitaxy (HVPE). ${ }^{12,13}$ Recently, we have studied $0.5 \mathrm{MeV}$ EI-induced deep traps in a control AlGaN/GaN SBD located about $3 \mathrm{~mm}$ away from the SBD mentioned above. In the control SBD, EI-induced trap $A_{2}$ can be clearly observed after consecutive EIs, as shown in Fig. 4(b). However, in this case, $A_{2}$ was found to appear on the shoulder of a negatively going DLTS signal, and thus accurate analysis is difficult. Such negatively going DLTS signals, as reported and discussed in Ref. 11, can possibly be related to holelike traps, but more study is needed on this issue. In any case, it is clear that trap $A_{2}$ can be observed in irradiated $\mathrm{AlGaN} / \mathrm{GaN}$ as well as in irradiated $\mathrm{GaN}$. Further study is also needed on the origin of the new trap $A_{e}$.

To study the effect of EI on the shallow traps at low temperatures, we use the irradiated SBD and the control SBD. For comparison, an earlier GaN SBD fabricated on a thick HVPE-grown GaN layer and irradiated with $0.7 \mathrm{MeV}$ electrons (using a dose of $5 \times 10^{15} \mathrm{~cm}^{-2}$ ) was also used. 

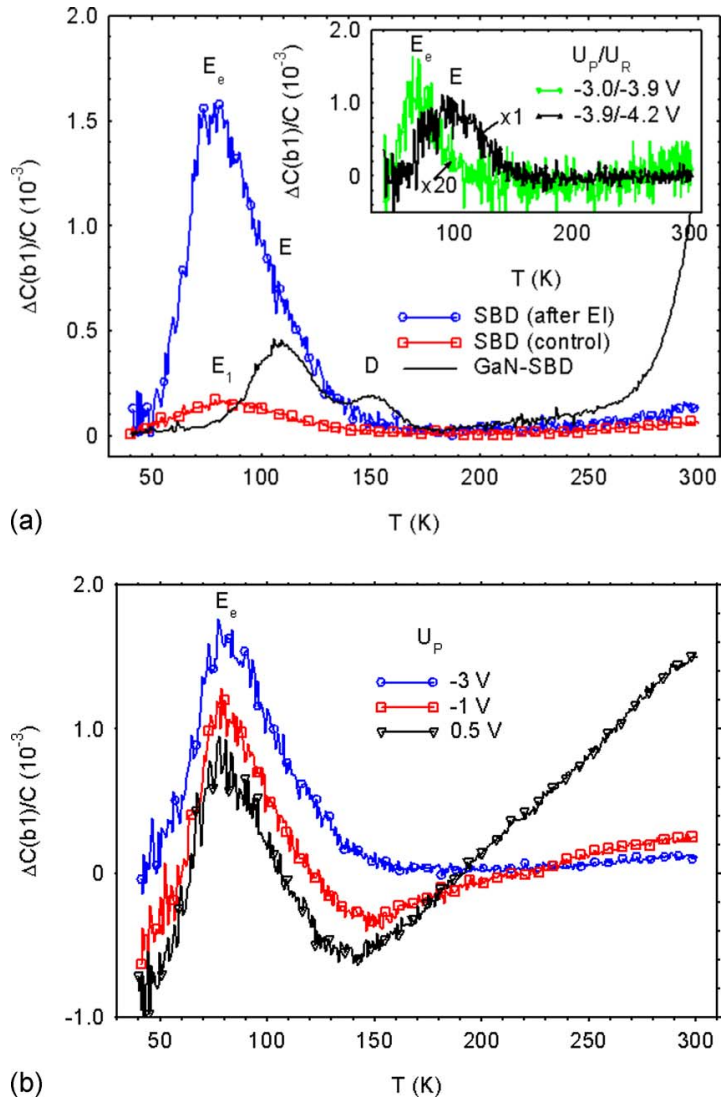

FIG. 5. (Color online) (a) Normalized DLTS spectra measured for irradiated and control AlGaN/GaN SBDs and a GaN SBD (for comparison). Conditions: $U_{P} / U_{R}=-3 /-4.1 \mathrm{~V}$ for SBD (after EI), $U_{P} / U_{R}=-3 /-4 \mathrm{~V}$ for SBD (control), and $U_{P} / U_{R}=0.4 /-3 \mathrm{~V}$ for GaN SBD, $T_{W}=20.5 \mathrm{~ms}$, and $t_{P}$ $=0.3 \mathrm{~ms}$. (Inset shows observation of $E_{e}$ and $E$ in different regions.) (b) Normalized DLTS spectra measured with different filling pulse heights $\left(U_{P}\right)$ from -3 to $0.5 \mathrm{~V}$ for the irradiated $\mathrm{AlGaN} / \mathrm{GaN}$ SBD. Conditions: $U_{R}=$ $-4.1 \mathrm{~V}, U_{P}=-3-0.5 \mathrm{~V}, T_{W}=20.5 \mathrm{~ms}$, and $t_{P}=0.3 \mathrm{~ms}$.

Normalized DLTS spectra at low temperatures from 40 to $300 \mathrm{~K}$, measured at $U_{R}=-4.1$ or $-4 \mathrm{~V}$ and $U_{P}=-3 \mathrm{~V}$ for the AlGaN/GaN SBDs in the pinch-off region and at $U_{R}=$ $-3 \mathrm{~V}$ and $U_{P}=0.4 \mathrm{~V}$ for the GaN SBD over the whole depletion region, are pictured in Fig. 5. From the figure, we see that (i) trap $E_{1}$ in the control SBD is an as-grown trap in the GaN buffer layer; (ii) in the irradiated SBD, there is an EI-induced trap $E_{e}$ with a density of one order of magnitude higher than that of trap $E_{1}$; and (iii) in the irradiated $\mathrm{GaN}$ $\mathrm{SBD}$, there are a pre-existing trap $D(0.25 \mathrm{eV})$ and an EIinduced trap $E$ with a density lower than that of $E_{e}$. However, when DLTS measurements were performed on the SBD with $U_{P} / U_{R}$ set in two different regions, i.e., in the $\mathrm{GaN}$ buffer (or pinch-off) region $\left(U_{P} / U_{R}=-3.9 /-4.2 \mathrm{~V}\right)$ and in part of the $2 \mathrm{DEG}$ region $\left(U_{P} / U_{R}=-3.0 /-3.9 \mathrm{~V}\right)$, we observed trap $E$ in the GaN buffer region and trap $E_{e}$ in a part of the 2DEG region [as shown in the inset of Fig. 5(a)]. The trap $E$ observed in the irradiated $\mathrm{AlGaN} / \mathrm{GaN}$ is quite similar to the trap $E$ observed in irradiated GaN. As shown in Fig. 5(b), trap $E_{e}$ can be observed in the DLTS spectra measured at $U_{P}$ changing from -3 to $0.5 \mathrm{~V}$, which corresponds to trap detection across the 2DEG region and near the surface of the AlGaN layer. Due to base line changes (possibly associated with holelike traps), the peak of $E_{e}$ was reduced as $U_{P}$ was

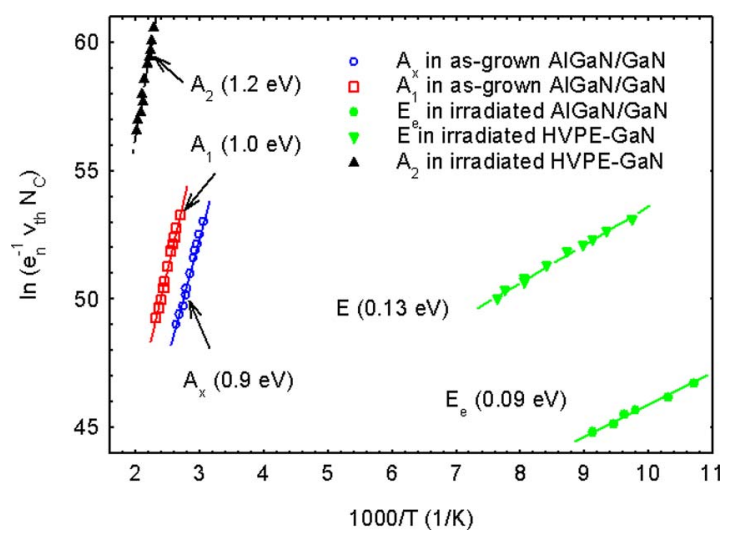

FIG. 6. (Color online) Arrhenius plots of $\ln \left(e_{n}{ }^{-1} V_{\text {th }} N_{C}\right)$ for major traps in as-grown and irradiated $\mathrm{AlGaN} / \mathrm{GaN}$ and GaN SBDs.

increased. The results indicate that an EI-induced shallow trap $E_{e}$ can be created in both the $2 \mathrm{DEG}$ region and the $\mathrm{AlGaN}$ barrier layer, as well as on the surface of the heterostructure.

To compare major traps in the as-grown and irradiated $\mathrm{AlGaN} / \mathrm{GaN}$ and $\mathrm{GaN}$ SBDs, Arrhenius plots of $\ln \left(e_{n}^{-1} V_{\text {th }} N_{C}\right)$ for traps $A_{2}, A_{1}, A_{x}, E$, and $E_{e}$ are shown in Fig. 6. Traps $A_{x}$ and $A_{1}$ were measured in two as-grown $\mathrm{AlGaN} / \mathrm{GaN}$ SBDs (one of them is used in this study), while traps $A_{2}$ and $E$ were measured in the irradiated HVPE-GaN $\mathrm{SBD}$, and trap $E_{e}$ was measured in the irradiated AlGaN/ GaN SBD. In comparison, we can see that (i) $A_{x}$ and $A_{1}$ have similar activation energies $\left(E_{T}=0.9 \mathrm{eV}\right.$ versus $\left.1.0 \mathrm{eV}\right)$ and capture cross sections $\left(\sigma_{n}=1.9 \times 10^{-10} \mathrm{~cm}^{2}\right.$ versus 4.4 $\times 10^{-11} \mathrm{~cm}^{2}$ ); (ii) as compared to $A_{x}$ or $A_{1}, A_{2}$ has higher $E_{T}(1.2 \mathrm{eV})$ and smaller $\sigma_{n}\left(1.4 \times 10^{-12} \mathrm{~cm}^{2}\right)$; and (iii) as compared to $E\left(E_{T}=0.13 \mathrm{eV}\right.$ and $\left.\sigma_{n}=1.3 \times 10^{-17} \mathrm{~cm}^{2}\right), E_{e}$ has lower $E_{T}(0.09 \mathrm{eV})$ and larger $\sigma_{n}\left(3.9 \times 10^{-16} \mathrm{~cm}^{2}\right)$. A further difference among the three deep traps is that $A_{x}$ or $A_{1}$ observed in as-grown $\mathrm{GaN}$ or $\mathrm{AlGaN} / \mathrm{GaN}$ SBDs shows an association with extended defects and less influence by EI, while $A_{2}$, not as prevalent in as-grown SBDs, shows a clear increase in irradiated $\mathrm{GaN}$ or $\mathrm{AlGaN} / \mathrm{GaN}$. However, by further comparing shallow traps in the irradiated AlGaN/GaN and GaN SBDs, there are at least three differences, i.e., (i) activation energy ( $0.09 \mathrm{eV}$ for $E_{e}$ versus $0.13 \mathrm{eV}$ for $E$ ), (ii) production rate (higher in the heterostructure than in the $\mathrm{GaN}$ layer), and (iii) electron capture behavior. As to item (iii), we observe a peak shift to lower temperatures, with increasing $t_{P}$ from 0.3 to $30 \mathrm{~ms}$, for trap $E$ in the irradiated GaN SBD but not for trap $E_{e}$ in the irradiated $\mathrm{AlGaN} / \mathrm{GaN} \mathrm{SBD}$, as shown in Fig. 7. The peak shift with increasing $t_{P}$ is due to the existence of two components in trap $E$, with different capture cross sections, as discussed in our earlier study. ${ }^{14}$ These differences might be related to the presence of a high electricfield in the $2 \mathrm{DEG}$ region of the $\mathrm{AlGaN} / \mathrm{GaN}$ heterostructure.

\section{SUMMARY AND CONCLUSIONS}

In summary, effects of $1 \mathrm{MeV}$ EI at room temperature on the electrical properties of $\mathrm{AlGaN} / \mathrm{GaN}$ heterostructures have been investigated using SBDs fabricated on AlGaN. The SBDs, before and after EI, were characterized by tempera- 


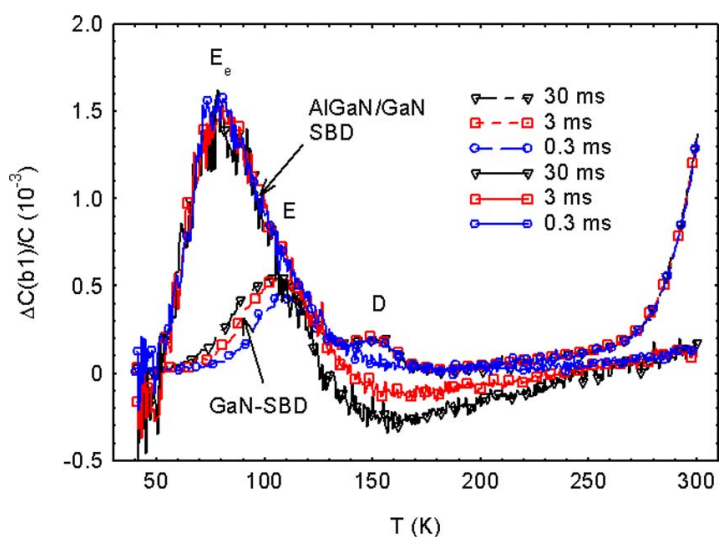

FIG. 7. (Color online) Comparison between EI-induced shallow traps in AlGaN/GaN and GaN SBDs. Conditions: $U_{P} / U_{R}=-3 /-4.1 \mathrm{~V}$ for AlGaN/ $\mathrm{GaN} \mathrm{SBD}$ and $U_{P} / U_{R}=0.4 /-3 \mathrm{~V}$ for $\mathrm{GaN} \mathrm{SBD}, T_{W}=20.5 \mathrm{~ms}$, and $t_{P}$ $=0.3-30 \mathrm{~ms}$.

ture dependent $I-V$ and $C-V$ and DLTS measurements. It is found that the irradiation causes (i) significant increases in leakage currents, dominated by tunneling conduction, for both reverse and low-forward biases; (ii) a negative shift in threshold voltage in the pinch-off region; and (iii) the creation of traps $A_{e}(\sim 1.1 \mathrm{eV})$ or $A_{2}(1.2 \mathrm{eV})$ and $E_{e}(0.09 \mathrm{eV})$ in the $\mathrm{GaN}$ buffer and $\mathrm{AlGaN}$ regions. These irradiationinduced traps, especially the shallow traps, can account for the increase in leakage currents and the shift in the threshold voltage in favor of the TSB model. However, as compared to traps $A_{2}(1.2 \mathrm{eV})$ and $E(0.13 \mathrm{eV})$, induced in thick GaN layers by EI, traps $A_{e}$ and $E_{e}$ in the irradiated AlGaN/GaN heterostructures show differences in activation energy and electron capture behavior. Further study is needed to understand the origin of the new trap $A_{e}$ and the reasons for the strong negatively going signals in the control SBD.

\section{ACKNOWLEDGMENTS}

We would like to thank to Dr. Donald Dorsey for encouragement and support. The work of Z.-Q.F., B.C., and D.C.L. was supported by AFRL under Contract No. FA8650-06-D5401 (monitored by D. Silversmith).

${ }^{1}$ X. Hu, B. K. Choi, H. J. Barnaby, D. M. Fleetwood, R. D. Schrimpf, S. Lee, S. Shojah-Ardalan, R. Wilkins, U. K. Mishra, and R. W. Dettmer, IEEE Trans. Nucl. Sci. 51, 293 (2004).

${ }^{2}$ G. Sonia, E. Richter, F. Brunner, A. Denker, R. Lossy, M. Mai, F. Lenk, J. Bundesmann, G. Pensl, J. Schmodt, U. Zeimer, L. Wang, K. Baskar, M. Weyers, J. Würfl, and G. Tränkle, Solid-State Electron. 52, 1011 (2008).

${ }^{3}$ J. W. McClory, J. C. Petrosky, J. M. Sattler, and T. A. Jarzen, IEEE Trans. Nucl. Sci. 54, 1946 (2007).

${ }^{4}$ A. Y. Polyakov, N. B. Smirnov, A. V. Govorkov, A. V. Markov, S. J. Pearton, A. M. Dabiran, A. M. Wowchak, B. Cui, A. V. Osinsky, P. P. Chow, N. G. Kolin, V. M. Boiko, and D. I. Merkurisov, Appl. Phys. Lett. 93, 152101 (2008).

${ }^{5}$ D. S. Green, S. R. Gibb, B. Hosse, R. Vetury, D. E. Grider, and J. A. Smart, J. Cryst. Growth 272, 285 (2004).

${ }^{6}$ H. Zhang, E. J. Miller, and E. T. Yu, J. Appl. Phys. 99, 023703 (2006).

${ }^{7}$ T. Hashizume, J. Kotani, and H. Hasegawa, Appl. Phys. Lett. 84, 4884 (2004).

${ }^{8}$ J. Kotani, M. Tajima, S. Kasai, and T. Hashizume, Appl. Phys. Lett. 91, 093501 (2007)

${ }^{9}$ P. Kordoš, D. Donoval, M. Florovič, J. Kováč, and D. Gregušová, Appl. Phys. Lett. 92, 152113 (2008).

${ }^{10}$ Z.-Q. Fang, D. C. Look, and L. Polenta, J. Phys.: Condens. Matter 14, 13601 (2002).

${ }^{11}$ Z.-Q. Fang, D. C. Look, D. H. Kim, and I. Adesida, Appl. Phys. Lett. 87, 182115 (2005)

${ }^{12}$ Z.-Q. Fang, G. Farlow, B. Claflin, and D. C. Look, Proceedings of 13th International Conference on Semiconducting and Insulating Materials, Beijing, China, edited by Z. G. Wang, Y. H. Chen, and X. L. Ye (IEEE, Piscataway, NJ, 2004), pp. 29-36.

${ }^{13}$ D. C. Look, Z.-Q. Fang, and B. Claflin, J. Cryst. Growth 281, 143 (2005).

${ }^{14}$ L. Polenta, Z.-Q. Fang, and D. C. Look, Appl. Phys. Lett. 76, 2086 (2000). 